Running head: Digital role modelling and parenting styles

\title{
Early childhood technoference threat is predicted by authoritative parenting, but not parental knowledge of digital risks.
}

\author{
Alistair Turvill $^{1 *}$, Dean Fido ${ }^{1}$, Emma Parker $^{1}$
}

${ }^{1}$ University of Derby (UK)

* Correspondence concerning this article should be addressed to Mr. Alistair Turvill, University of Derby, Kedleston Road, Derby, DE22 1GB, UK. Tel: (01332) 592628. Email: A.Turvill@derby.ac.uk.

\section{Declaration of Competing Interests}

The author(s) declare no potential competing or financial interests with respect to the research, authorship, and/or publication of this article.

\section{Funding}

This work was supported by an Undergraduate Research Scholarship Scheme (URSS) fund (£230) from University of Derby. 


\section{Acknowledgement}

The authors take responsibility for the integrity of the data, the accuracy of the data analyses, and have made every effort to avoid inflating statistically significant results. Data transparency is important to the authors and as such our data and analyses were made open access prior to peer review. 


\begin{abstract}
Frequent disruption to parent-child interactions by technology (technoference) significantly impacts problematic behaviours and socioemotional development. Currently unknown is whether this is a novel challenge presented to all parents by the proliferation of technology, or representative of existing maladaptive parenting practices. Twohundred and fifteen UK parents $\left(M_{\text {age }}=36\right.$ years $)$ self-reported parenting style (i.e., authoritative, authoritarian, permissive), technoference, problematic technology use, and perception of digital risks. Results indicated higher authoritative parenting styles predicted reduced technoference $(b=-.29)$, and problematic technology use was associated with increased technoference and higher use of both permissive and authoritarian parenting styles. For the first time, prevalence of technoference is shown to disproportionately threaten a subset of children. Supplementary materials:

[https://osf.io/b6rn3/?view_only=5b422beef2ef4aea95c28e28e55f1a5e]
\end{abstract}

Key words: Technoference, Early childhood, Parenting styles, Digital risk 


\section{Early childhood technoference threat is predicted by Authoritative parenting, but not parental knowledge of digital risks}

Technology is omnipresent in Western societies, epitomised by the proliferation of smartphone, tablet, and wearable technologies over the past decade (Ofcom, 2018). This development is observable in UK families, with $52 \%$ of children aged 3-4 spending an average of 9 hours a week online, and children aged 5-7 spending 30+ hours across all devices (Ofcom, 2019). Middleton (2007) describes the "always on" environment that technology creates for parents, and its potential for problematic (McDaniel \& Radesky, 2018b) or compulsive use (Oulasvirta, Rattenbury, Ma, \& Raita, 2012) with parents trying to 'keep-up' with developments (Zimmerle, 2019). Evidence below presents the impact of these factors on children.

\section{Parents' own technology behaviours}

Children commonly observe adults using technologies (Lauricella, Wartella, \& Rideout, 2015), and consistent with Bandura's (1978) Social Learning Theory, imitate or adopt observed behaviours (Lauricella et al., 2015). Increased parental screen-time is associated with greater child exposure to media (Jago et al., 2012) and use of screens (Lauricella et al., 2015); resulting in reduced quantities of parent-child interactions (McDaniel \& Radesky, 2018b). Termed “technoference” (McDaniel, 2015), technological interruptions to daily parent-child interaction are positively associated with parents' problematic technology use (McDaniel \& Radesky, 2018b).

$89 \%$ of parents report interruption to child interactions due to technology (McDaniel \& Radesky, 2018a), with smartphones a common distraction (Beamish et al., 2019). 
Specifically, Radesky et al. (2015) find reductions in verbal and non-verbal interactions between mothers and children, due to repeated maternal device checking. Further, Hiniker et al. (2015) report using technology around young children is associated with reduced responsivity to child-bids for attention. The importance of parent-child interactions in driving developmental processes (Gadaire et al., 2017) means frequent technoference can negatively impact development. Evidence shows technoference impacts children's socio-emotional and behavioural development (Stockdale et al., 2018), and is associated with greater problematic externalising and internalising behaviours (McDaniel \& Radesky, 2018a, McDaniel \& Radesky, 2018b).

Parental knowledge and mediation of child behaviour

Parents set boundaries regarding type or amount of technology use allowed by children, determining their degree of exposure to digital threat. Actions such as these are commonly interpreted with respect to established behavioural frameworks, for example Barkin, Richardson, Klinepeter, Finch, and Krcmar's (2006): 'Mediation Strategies', consisting of: Restrictive; limiting access to devices or content, instructive; adopting couse to guide and monitor use, and unlimited; presenting a child with unfettered access to technology (and the highest level of risk). Similarly Baumrind's (1971) Parenting Dimensions, with; high levels of controlling or indulgent behaviours are associated with "authoritarian" and "permissive" parenting styles respectively, whereas actions seen as having warmth, high responsiveness, and clear boundaries are associated with an “authoritative" style (Lamborn et al., 1991). 
Although not derived from Baumrind (1971), Barkin et al.'s (2006) 'strategies' are analogous to these, evidenced by similar findings with current research: Barkin et al.'s (2006) report significant relations between deficits in parental knowledge and use of maladaptive unlimited mediation strategies. Similarly, September, Rich, \& Roman, $(2016,2017)$ show parents with poorer understanding of child development make greater use of maladaptive authoritarian strategies. The present study aims to build on these; establishing whether parental perceived knowledge of digital risk is associated with particular parenting strategies or technoference. Livingstone \& Helsper (2008) believe there is a high potential for the adoption of maladaptive parenting practices concerning technology due to lack of knowledge. Further, Wong et al. (2015) found just 1 in 8 parents felt confident protecting their children online, with a lack of parental knowledge regarding the use of technology affecting confidence in supervising their children. understanding in this area is important for furthering understanding factors of impacting childhood exposure to digital risk.

\section{The present study}

While technoference is broadly understood, it remains unknown whether it is a novel, emerging phenomenon fostered by technological expansion and present in most families. Or, whether it is behaviour predominantly associated with wider maladaptive parenting practices; potentially limited to a subset of families. Similarly, unknown is whether parental perception of digital risk is associated with technoference or parenting styles. This study will explore relationships between parenting styles, perception of digital risk, problematic technology use, and levels of technoference, including whether levels of technoference can be predicted by parenting style, or knowledge of risk. The expectation is that technological expansion will continue to permiate into daily lives (Williams, 
2018), greater understanding of technoference within UK families is essential to reducing the risks posed by technology to children.

\section{Methods}

\section{Participants}

Assuming an anticipated medium effect size (ensuring the practical importance of observed effects) and an adjusted alpha level of .016 to account for multiple testing between parenting styles, an a priori power analysis (G*Power, v3.1.9.2) determined a minimum of 168 participants were required to have $95 \%$ power in the planned analyses. A total of 228 participants responded to an online questionnaire advertised through the crowdsourcing website Prolific. Removing cases where inclusion criteria were not met ( $n$

$=1$ ), and where incomplete ( $>5 \%$ of the data missing) or inconsistent responses were present $(n=5)$, data from 223 participants were analysed $\left(M_{\text {age }}=36.04\right.$ years, $S D=6.30$; $46.2 \%$ male, $84 \%$ married to or living with partner). Inclusion criteria required that participants were fluent in English, aged at least 18 years, and have at least one child within the Early Childhood Development age range ( 0 - 8 years) due to interruptions within this period being associated with greater developmental impact (World Health Organization, n.d.). Participants provided written informed consent in accordance with approved central university research protocols and national ethical guidelines by ticking a box on both the first and last pages of the survey. All completers were reimbursed with $£ 0.85$ for participation. It will focus solely on UK parents to avoid confounds arising from cultural variation regarding parenting style (Nadal, 2017) and differences in both access to, and social norms regarding technology (Lee, Trimi, \& Kim, 2013).

\section{Materials}


Demographics. Participants were asked to report their age, sex, marital status, number of children, and their respective ages and sexes.

\section{Technology Device Interference Scale (TDIS; Adapted from McDaniel \&}

Coyne, 2016, and McDaniel \& Radesky, 2018b). Participants were asked to report how often their common child-parent events (meal times, bed-time, morning routine, and outings) are interrupted by technologies. Responses are recorded on a 6-point Likert scale ranging from "Never" to "All the time". An additional option of "Not typically present for this activity"' was also included for parents who are not able to engage in certain events due to other commitments. In such instances, mean scores were created with these items omitted. Higher scores indicated greater technoference. Alpha scores for this and other scales for our sample are reported in Table 3.

\section{Parenting Styles \& Dimensions Questionnaire - Short Version (PSDQ-SV;}

Robinson, Mandleco, Oslen, \& Hart, 2001). The PSDQ-SV comprises 32 -items that measure the propensity to parent in a given style using a 5-point scale anchored from "Never" to "Always". The scale can be used to define three parenting styles; authoritative (e.g., "Gives praise when child is good."), authoritarian (e.g., "Slaps child when the child misbehaves.”), and permissive (e.g., "Spoils child.”). High scores indicated greater presentation of parenting style.

\section{Parent Problematic Digital Technology Use (PTU; McDaniel \& Radesky,}

2018b). Self-perceived problematic technology use was assessed via a three-item measure, with responses recorded on a 6-point likert scale ranging from "Strongly disagree" "Strongly agree". Questions tapped into parents' ability to resist urges to 
check/use technology, whether they feel they use technology too much, and how preoccupied they are by thoughts related to technology (e.g., "I often think about calls or messages I might receive on my mobile phone."). Higher scores were indicative of more problematic technology use.

\section{Parents Perceptions of Digital Risk (PDR; Adapted from İnan-Kaya, Mutlu-} Bayraktar, \& Yllmaz, 2018). Originally a 10-item measure (reduced to 9 following our factor analysis), the PRD was adapted as a means of assessing parents' beliefs about their own understanding of digital and technological risks posed to children, as well as beliefs about whether they are able to meaningfully intervene if a threat is present (e.g., "I have knowledge of the possible harmful content that my child may face whilst surfing the internet."). Responses are reported on a 3-point scale anchored from "I have no knowledge" to "I have enough knowledge". Higher scores indicate greater self-perceived knowledge.

\section{Procedure}

The study was approved by an institutional ethical review panel prior to data collection (REMOVED FOR PEER REVIEW). After entering demographic information, participants were presented with the TDIS, PSDQ-SV, PTU, and PDR in a randomised order. Randomisation was conducted for each participant by the survey software to reduce the likelihood of order effects influencing the data. On average, the study took less than 10 minutes to complete.

\section{Analysis plan}


The nascent nature of the research topic meant there was a dearth of reliable measures available. Reported first are two principal axis factor analyses conducted on the 10-item PDR and 4-item TDIS in order to identify reliability, individual sub-scales, and redundant items. Second, Pearson correlations were computed between demographic variables (age, sex, and number of children), the focal predictors (authoritarian, authoritative, and permissive parenting styles), and the dependent variables (technoference, and selfreported knowledge of digital and technological risks posed to children). Finally, we conducted three hierarchical multiple regression analyses in order to identify unique predictors of the dependant variables noted above after controlling for demographic variables.

\section{Results}

Data Cleaning. Of the 223 participants, eight were excluded from all analyses except for the factor analyses due to incomplete parenting styles data $(>5 \%$ missing data), leaving a final sample of $n=215\left(M_{\text {age }}=35.90\right.$ years, $S D=6.35,45.6 \%$ male, $84 \%$ married to or living with partner).

Factor Analysis: PDR. Bartlett's test of sphericity was significant, $p<.001$, and the Kaiser-Meyer-Olkin measure verified the sampling adequacy for the analysis, $\mathrm{KMO}=$ .88 , with all KMO values for individual items greater than .80 , which is well above the acceptable limit of .50. An initial analysis was run to obtain eigenvalues for each factor in the data. Convergence of the scree plot (see supplementary material) and eigenvalues over Kaiser's criterion of 1, suggested a two factor structure, which combined explained $66.95 \%$ of the variance. Table 1 shows the factor loadings after rotation. The items that cluster on the same factor suggest that factor 1 represents 'External Threat' and factor 2 
represents 'Health Implications'. Owing to the equal loadings of Question 8, this item was removed from the questionnaire during subsequent analyses.

Table 1. Summary of exploratory factor analysis results for the PDR $(n=223)$

\begin{tabular}{|c|c|c|}
\hline & Factor 1 & Factor 2 \\
\hline $\begin{array}{l}\text { 4. I have knowledge of the risk that my child may be exposed to cyber } \\
\text { bullying. }\end{array}$ & .77 & \\
\hline $\begin{array}{l}\text { 7. I have knowledge of the potential risk that malicious individuals may } \\
\text { abuse my child while they are using digital media. }\end{array}$ & .70 & .38 \\
\hline $\begin{array}{l}\text { 9. I have knowledge of the risks of my child sharing his/her own photos and } \\
\text { videos on social media accounts such as Facebook, Snapchat, Instagram etc. } \\
\text { while using digital devices. }\end{array}$ & .69 & .42 \\
\hline $\begin{array}{l}\text { 1. I have knowledge of the risk that my child may share personal information } \\
\text { (address, identity, credit card etc.) with other individuals when online. }\end{array}$ & .68 & \\
\hline $\begin{array}{l}\text { 5. I have knowledge of the potential risks of my child sharing his/her location } \\
\text { while using digital devices. }\end{array}$ & .68 & .44 \\
\hline $\begin{array}{l}\text { 2. I have knowledge of the possible harmful content that my child may face } \\
\text { whilst surfing the internet. }\end{array}$ & .66 & \\
\hline $\begin{array}{l}\text { 3. I have knowledge of the potential risk that my child may be the perpetrator } \\
\text { or the victim of a crime committed by use of digital media. }\end{array}$ & .65 & \\
\hline $\begin{array}{l}\text { 10. I have knowledge of how frequent or prolonged use of technological } \\
\text { devices by my child may cause health problems. }\end{array}$ & & .83 \\
\hline $\begin{array}{l}\text { 6. I have knowledge of how frequent or prolonged use of technological } \\
\text { devices by my child may cause technological addiction. }\end{array}$ & & .77 \\
\hline $\begin{array}{l}\text { 8. I have knowledge of the potential risk that my child may be kidnapped by } \\
\text { strangers that he/she meets online. }\end{array}$ & .53 & .55 \\
\hline Eigenvalues & 5.47 & 1.23 \\
\hline$\%$ of variance & 54.70 & 12.25 \\
\hline$\alpha$ & .89 & .81 \\
\hline
\end{tabular}

Note. Items attributed to each factor appear in bold. Factor loadings $<.30$ have been suppressed.

Factor Analysis: TDIS. Bartlett's test of sphericity was significant, $p<.001$, and the Kaiser-Meyer-Olkin measure suggested reasonable sampling adequacy for the analysis, $\mathrm{KMO}=.57$. All $\mathrm{KMO}$ values for individual items greater than .56 , using an acceptable cut-off of .50. An initial analysis was run to obtain eigenvalues for each factor 
in the data. Convergence of the scree plot (see supplementary material) and eigenvalues over Kaiser's criterion of 1, suggested that activities whereby technoference can occur can be treated as a single factor. This factor explained $46.44 \%$ of the variance (see Table 2 for factor loadings).

Table 2. Summary of exploratory factor analysis results for the TDIS $(n=223)$

\section{Factor 1}

2. Meal times.

.73

4. Bedtime routine. .53

1. Morning routine. .50

3. Outings. .39

Eigenvalues 1.86

$\%$ of variance 46.44

$\alpha$ .61

Sex differences. Means and standard deviations for questionnaire data are reported in Table 3. Independent $t$-tests were used to delineate sex differences within our sample. Females reported greater levels of authoritative parenting styles, $t(213)=-3.49, p$ $=.001, d=.40$, and males reported greater levels of authoritarian, $t(213)=2.19, p=.03$, $d=.27$, and permissive, $t(213)=2.02, p=.04, d=.28$, parenting styles. There were no significant differences in age, number of children, knowledge (neither external threats nor health implications), technoference, instances of interruption as a result of technology, or PTU. 
Correlation analyses. Scores on technoference and knowledge pertaining to digital risks were negatively skewed, suggesting the majority of parents professed to having a proficient knowledge of digital risks and were also aware of their own problematic technology use. Nevertheless, as this can be considered representative of the general population, Pearson correlations between predictor and dependent variables were computed (see Table 4). Age was positively correlated with number of children, and each factors of the PDR (knowledge of external threats and knowledge of health implications) were positively correlated with one another as well as the total PDR score. Technoference was positively associated with the self-reported number of times a day parents reported technology interrupting their interactions, and both variables were positively associated with perceptions of own problematic technology use and authoritative, but not authoritarian or permissive parenting styles. Authoritarian parenting styles were associated with both authoritative (negatively) and permissive (positively) parenting styles.

\section{[Table 4. Around here]}

Regression analyses. We conducted three hierarchical multiple regression analyses. In each analysis, age, number of children, and sex were entered at stage one, and authoritative, authoritarian, and permissive parenting styles were entered at stage two. The dependant variables were scores on the TIDS as well as the knowledge of external threats and knowledge of health implications facets of the PDR. Model one met the assumptions required for hierarchical multiple regression analysis, however models two and three did not on account of their negative skew towards maximum perceived knowledge of external threats and health implications. In an attempt to resolve this issue, models two and three underwent robust bootstrapping (Field, 2018). 
Model one: TIDS. At stage one, the variables of age, sex, and number of children did not contribute significantly to the regression model, $F(3,211)=.86, p=.46$; accounting for only $1.2 \%$ of the variation in technoference. Introducing authoritative, authoritarian, and permissive parenting styles explained an additional $6.1 \%$ of variation in technoference totalling $7.3 \%$, and this $R^{2}$ was significant, $F(3,208)=4.53, p=.004$. The only significant predictor was authoritative parenting style, such that when holding other parenting styles constant, a unit increase in authoritative parenting would lead to a .29 unit decrease in technoference, $b=-.29(\mathrm{SE}=.11), t=-2.53, p=.01$. Model coefficients are presented in Table 5.

Table 5. Linear model of predictors of TDIS score.

\begin{tabular}{lccccccc}
\hline \multicolumn{1}{c}{ Variable } & $b(\mathrm{SE})$ & $\beta$ & $\mathrm{B} 95 \% \mathrm{CI}$ & $t$ & $p$ & $\mathrm{R}^{2}$ & $\Delta \mathrm{R}^{2}$ \\
\hline Step 1 & & & & & .46 & .01 & -.002 \\
Age & $.01(.01)$ & .09 & {$[-.01, .03]$} & 1.35 & .18 & & \\
Sex & $.08(.11)$ & .05 & {$[-.14, .29]$} & .71 & .48 & \\
Number of children & $.02(.06)$ & .02 & {$[-.10, .14]$} & .32 & .75 & \\
Step 2 & & & & & .004 & .07 & .05 \\
Age & $.02(.01)$ & .12 & {$[-.002, .03]$} & 1.74 & .08 & & \\
Sex & $.16(.11)$ & .10 & {$[-.06, .37]$} & 1.40 & .16 & & \\
Number of children & $.003(.06)$ & .003 & {$[-.11, .12]$} & .05 & .96 & & \\
Authoritative & $-.29(.11)$ & -.18 & {$[-.52,-.06]$} & -2.53 & .01 & & \\
Authoritarian & $.25(.13)$ & .15 & {$[-.01, .51]$} & 1.87 & .06 & & \\
Permissive & $-.22(.14)$ & -1.58 & {$[-.50, .06]$} & -1.58 & .12 & \\
\hline
\end{tabular}


Model two: Knowledge of external threats. At stage one, the variables of age, sex, and number of children did not contribute significantly to the regression model, $F(3,210)$ $=.28, p=.84$; accounting for only $.4 \%$ of the variation in technoference. Introducing authoritative, authoritarian, and permissive parenting styles explained an additional .9\% of variation in technoference totalling $1.3 \%$; this $R^{2}$ was also not significant, $F(3,207)=$ $.62, p=.61$.

Model three: Knowledge of health implications. At stage one, the variables of age, sex, and number of children did not contribute significantly to the regression model, $F(3$, $210)=.64, p=.59$; accounting for $1 \%$ of the variation in technoference. Introducing authoritative, authoritarian, and permissive parenting styles explained an additional .5\% of variation in technoference totalling $1.4 \%$; this $R^{2}$ was also not significant, $F(3,207)=$ $.35, p=.79$.

\section{Discussion}

Results indicated that authoritative, but not authoritarian or permissive parenting styles predicted technoference. There were no significant sex differences for demographics, however, differences were found regarding parenting styles; with fathers more likely to adopt both authoritarian and permissive approaches, and mothers more disposed to authoritative (findings in this area are inconsistent, see Nadal, 2017).

\section{Parents' behaviours}

Technoference was measured via the TDIS, analysis of which evidenced a one-factor solution and moderate levels of reliability (.61; Taber, 2018). Convergent support for the measure's validity is seen in significant correlations with PTU (supporting findings from 
McDaniel and Radesky, 2018b), and self-reported number of technology interruptions. The TDIS measure presented subjective response categories (never, always, etc) avoiding imposition of numerical limits on participants, however, strong correlations between responses on TDIS and self-reported numerical mean number of daily interruptions suggests that there is agreement regarding approximately how many times labels such as 'very often' represent.

Findings show parents with higher PTU scores make significantly greater use of permissive parenting styles, indicating the potential for children of these parents to experience lower levels of mediation and higher levels of risk (Barkin et al., 2006). Significant contrasting relationships were found between parenting styles, with strong positive correlations between permissive and authoritarian strategies, and significant negative correlations with both and authoritative approaches. This implies parents are somewhat typological and consistent in their behaviours, inturn presenting children with consistently more positive or negative parenting approaches. However, although not explained via detailed inspection of the data and output, it should be noted that at .12 , the reliability for the permissive subscale measure was low (Taber, 2018) and so inferences from this subscale should be made cautiously.

Novelly; the level of authoritarian parenting reported by participants, significantly predicted the level of technoference, with greater adoption of these strategies associated with significantly lower incidence. For the first time it can be said that the prevalence of technoference in childhood is not uniform, rather, some children are more threatened than others. Furthermore, with some parents reporting significantly higher PTU, permissive parenting, and technoference, this creates increased opportunity for developmental 
displacement and reduced mediation. The additional social influence parents have upon their children should be reiterated, with high potential for children to adopt the behaviours of their parents (Bandura, 1978).

\section{Parents' Knowledge:}

No significant relationship between parenting styles and the perception of digital risk were found, with scores here notably high such that a majority of parents reported they knew enough about a broad range of digital threats. Parents who scored highly on factor 1 (external threats) were significantly more likely to also score highly on factor 2 (health implications). This finding conflicts with previous studies showing differences between certain parenting styles and knowledge (September et al., 2016, 2017; Wong et al., 2015), perhaps representing cultural variation between populations. However, the aforementioned skewing of responses to the higher end of the scale might also indicate a need to expand the response options available. Additionally, including some form of knowledge testing (as was the case in September et al., 2016, 2017) to support the veracity of self-reported participant knowledge would provide additional insight (Rosenman, Tennekoon, \& Hill, 2011).

\section{Limitations}

Measures used within this manuscript have been derived from peer reviewed literature, however current validity estimates of some areas suggest capacity for further improve upon robustness and specificity of these measures. Future research might refine these instruments for further investigation. Additionally, the use of self-report measures carries a high potential for bias (Rosenman, et al., 2011). This study only recruited UK participants, presenting opportunity to expand investigation to other cultures and 
populations. As the present study focuses on how levels of technoference vary with regard to parenting style, it was legitimate to limit recruitment to online methods, however broadening of the current study findings could seek to diversify this aspect.

\section{Conclusion}

This study reports on a varied sample including single and co-parenting families, a balanced sex profile, and a range of ages. While the rise of technology in western societies has been broadly ubiquitous in all aspects of life, technoference does not appear to be a novel threat that presents equally in all UK families. Rather, a subset of children is disproportionately exposed, with a potential for significant impact on developmental trajectories. There were no significant relationships between parenting style and PDR, conflicting with previous studies and future research should seek to investigate this finding via refined methods. Future work may consider whether measures of parenting style can be used to help identify families that may be more threatened by technology. Evidence suggests that maladaptive parenting practices can be adjusted with well targeted and appropriate support (Peter, 2018), suggesting childhood digital risk can be reduced. 


\section{References}

Baumrind, D. (1971). Current patterns of parental authority. Developmental Psychology, 4(1), 1-103.

Barkin, S., Ip, E., Richardson, I., Klinepeter, S., Finch, S., \& Krcmar, M. (2006). Parental media mediation styles for children aged 2 to 11 years. Archives of Pediatrics \& Adolescent Medicine, 160(4), 395-401.

Bandura, A. (1978). Social learning theory of aggression. Journal of Communication, $28(3), 12-29$.

Beamish, N., Fisher, J., \& Rowe, H. (2019). Parents' use of mobile computing devices, caregiving and the social and emotional development of children: a systematic review of the evidence. Australas Psychiatry, 27(2), 132-143.

Field, A. P. (2018). Discovering statistics using SPSS. Thousand Oaks, California: SAGE Publications.

Gadaire, D. M., Henrich, C. C., \& Finn-Stevenson, M. (2017). Longitudinal Effects of Parent-Child Interactions on Children's Social Competence. Research on Social Work Practice, 27(7), 767-778.

Hiniker, A., Sobel, K., Suh, H., Sung, Y. C., Lee, C. P., \& Kientz, J. A. (2015). Texting while parenting: How adults use mobile phones while caring for children at the playground. Paper presented at the 33rd Annual ACM Conference on Human Factors in Computing Systems, ACM, 727-736.

İnan-Kaya, G., Mutlu-Bayraktar, D., \& Y1lmaz, Ö. (2018). Digital parenting: Perceptions on digital risks. Kalem Uluslararası Eğitim ve İnsan Bilimleri Dergisi, 137-163.

Jago, R., Stamatakis, E., Gama, A., Carvalhal, I. M., Nogueira, H., Rosado, V., \& Padez, C. (2012). Parent and child screen-viewing time and home media environment. American Journal of Preventive Medicine, 43(2), 150-8.

Lamborn, D., Mounts, S., Steinberg, L., Eastin, M., \& Dornbusch, M. (1991). Patterns of competence and adjustment among adolescents from authoritative, authoritarian, indulgent, and neglected families. Child Development, 62(5), 1049-1065.

Lauricella, A. R., Wartella, E., \& Rideout, V. J. (2015). Young children's screen time: The complex role of parent and child factors. Journal of Applied Developmental Psychology, 36, 11-17.

Lee, S. G., Trimi, S., \& Kim, C. (2013). The impact of cultural differences on technology adoption. Journal of World Business, 48(1), 20-29.

Livingstone, S. \& Helsper, E. J. (2008). Parental mediation of children's Internet use. Journal of Broadcasting \& Electronic Media, 52(4), 581- 599. 
McDaniel, B. T. (2015). "Technoference": Everyday intrusions and interruptions of technology in couple and family relationships. In C. J. Bruess (Ed.), Family communication in the age of digital and social media. New York: Peter Lang Publishing.

McDaniel, B. T., \& Coyne, S. M. (2016). "Technoference": The interference of technology in couple relationships and implications for women's personal and relational well-being. Psychology of Popular Media Culture, 5(1), 85.

McDaniel, B. T. \& Radesky, J. S. (2018a). Technoference: longitudinal associations between parent technology use, parenting stress, and child behaviour problems. Pediatric Research, 84, 210-218.

McDaniel, B. T. \& Radesky, J. S. (2018b). "Technoference: Parent distraction with technology and associations with child behavior problems." Child Development, 89(1), 100-109.

Middleton, C. A. (2007). Illusions of balance and control in an always-on environment: A case study of BlackBerry users. Continuum: Journal of Media \& Cultural Studies, 21(2), 165-178.

Nadal, K. L. (Ed.). (2017). The SAGE encyclopedia of psychology and gender. SAGE Publications.

Ofcom. (2018). Communications Market Report 2018. Retrieved from: https://www.ofcom.org.uk/_data/assets/pdf_file/0022/117256/CMR-2018narrative-report.pdf.

Ofcom. (2019). Children and parents: Media use and attitudes report 2018. Retrieved from: https://www.ofcom.org.uk/_data/assets/pdf_file/0024/134907/Children-andParents-Media-Use-and-Attitudes-2018.pdf.

Oulasvirta, A., Rattenbury, T., Ma, L., \& Raita, E. (2012). Habits make smartphone use more pervasive. Personal and Ubiquitous Computing, 16(1), 105-114.

Peter, S. (2018). Research digest: parenting interventions and the role of parental factors and styles in child mental health. Journal of Child Psychotherapy, 44(2), 275-283.

Radesky, J., Miller, A., Rosenblum, K., Appugliese, D., Kaciroti, N., \& Lumeng, J. (2015). Maternal mobile device use during a structured parent-child interaction task. Academic Pediatrics, 15(2), 238-44.

Robinson, C. C., Mandelco, B., Olsen, S. F., \& Hart, C. H. (2001). The parenting styles and dimensions questionnaire (PSQD). In B. F. Perlmutter, J. Touliatos \& G. W. Holden (Eds.), Handbook of family measurement techniques: Vol. 3. Instruments \& Index (pp. 319-321). Thousand Oaks: Sage.

Rosenman, R., Tennekoon, V., \& Hill, L. G. (2011). Measuring bias in self-reported data. International Journal of Behavioural \& Healthcare Research, 2(4), 320-332. 
September, S. J., Rich, E. G., \& Roman, N. V. (2016). The role of parenting styles and socio-economic status in parents' knowledge of child development. Early Child Development and Care, 186(7), 1060-1078.

September, S. J., Rich, E. G., \& Roman, N. V. (2017). Association between knowledge of child development and parenting: A Systematic Review. The Open Family Studies Journal, 9(1).

Stockdale, L., Coyne, S., \& Padilla-Walker, L. (2018). Parent and Child Technoference and socioemotional behavioral outcomes: A nationally representative study of 10to 20-year-Old adolescents, Computers in Human Behavior, 88, 219-226.

Taber, K. S. (2018). The use of Cronbach's alpha when developing and reporting research instruments in science education. Research in Science Education, 48(6), 1273-1296.

World Health Organization, (n.d.). Early child development. Retrieved from: https://www.who.int/topics/early-child-development/en/

Williams, K.C., (2018). Intersections of Technology and Civil Society. Department for International Development. HM Government. Downloaded 14/8/2019 from: https://assets.publishing.service.gov.uk/media/5c6c257140f0b647ada07270/448_ T ech_and_Civil_Society_Nexus.pdf.

Wong, Y. C., Ho, K. M., \& Chen, H. (2015). Internet supervision and parenting in the digital age: The case of Shanghai. The Open Family Studies Journal, 7(1), 112-123.

Zimmerle, J. (2019). Limiting Technoference: Healthy Screen Time Habits for New Parents. International Journal of Childbirth Education, 34(2), 54-59. 
Table 3. Descriptive statistics for questionnaire scores with between sex comparisons.

\begin{tabular}{|c|c|c|c|c|c|}
\hline & $\alpha$ & $\begin{array}{l}\text { Total } \\
\text { M (SD) }\end{array}$ & $\begin{array}{c}\text { Males }(n=98) \\
\text { M (SD) }\end{array}$ & $\begin{array}{c}\text { Females }(n=117) \\
\text { M (SD) }\end{array}$ & $p$ \\
\hline Age & - & $35.90(6.35)$ & $36.11(6.51)$ & $35.88(6.27)$ & .65 \\
\hline Number of children & - & $1.92(.91)$ & $1.86(.86)$ & $1.98(.95)$ & .39 \\
\hline PDR - External Threats & .89 & $1.82(.34)$ & $1.81(.38)$ & $1.83(.32)$ & .80 \\
\hline PDR - Health Implications & .83 & $1.68(.51)$ & $1.63(.56)$ & $1.72(.47)$ & .16 \\
\hline PDR - Total & .89 & $1.75(.37)$ & $1.72(.41)$ & $1.78(.35)$ & .28 \\
\hline TDIS & .63 & $1.47 .79)$ & $1.43(.76)$ & $1.54(.83)$ & .49 \\
\hline Times interrupted & - & $3.77(3.34)$ & $3.72(3.06)$ & $3.81(3.60)$ & .85 \\
\hline PTU & .75 & $3.15(.94)$ & $3.11(.98)$ & $3.21(.94)$ & .50 \\
\hline Authoritative & .82 & $3.98(.50)$ & $3.86(.56)$ & $4.06(.42)$ & .001 \\
\hline Authoritarian & .74 & $2.08(.48)$ & $2.16(.51)$ & $2.03(.47)$ & .03 \\
\hline Permissive & .12 & $2.43(.44)$ & $2.49(.45)$ & $2.37(.41)$ & .04 \\
\hline
\end{tabular}

Note. $\mathrm{PDR}=$ perceptions of digital risk, TDIS $=$ technoference, $\mathrm{PTU}=$ problematic technology use.

Significant differences highlighted in bold. 
Table 4. Pearson correlations between variables for the whole sample.

\begin{tabular}{|c|c|c|c|c|c|c|c|c|c|c|c|}
\hline & 1 & 2 & 3 & 4 & 5 & 6 & 7 & 8 & 9 & 10 & 11 \\
\hline [1] Age & - & $.16^{*}$ & -.04 & -.01 & -.02 & .10 & .13 & -.10 & .11 & -.05 & -.04 \\
\hline [2] Number of children & & - & -.06 & .02 & -.01 & .04 & -.03 & .05 & -.04 & .10 & .08 \\
\hline [3] PDR - External Threats & & & - & $.51 * * *$ & $.81 * * *$ & -.06 & .01 & -.04 & .03 & .05 & -.02 \\
\hline [4] PDR - Health Implications & & & & - & $.92 * * *$ & -.07 & .004 & -.06 & .02 & .03 & -.04 \\
\hline [5] PDR - Total & & & & & - & -.07 & .01 & -.06 & .03 & .04 & -.04 \\
\hline [6] TDIS & & & & & & - & $.49 * * *$ & $.41 * * *$ & $-.12 * *$ & .11 & -.06 \\
\hline [7] Times interrupted & & & & & & & - & $.27 * * *$ & $-.17 *$ & .06 & -.004 \\
\hline [8] PTU & & & & & & & & - & -.04 & .10 & $.15^{*}$ \\
\hline [9] Authoritative & & & & & & & & & - & $-.24 * * *$ & -.03 \\
\hline [10] Authoritarian & & & & & & & & & & - & $.50 * * *$ \\
\hline [11] Permissive & & & & & & & & & & & - \\
\hline
\end{tabular}

Note. $\mathrm{PDR}=$ perception of digital risk, TDIS $=$ technoference, $\mathrm{PTU}=$ problematic technology use. ${ }^{*} p<.05,{ }^{* *} p<.01, * * * p<.001$. 